\title{
Rising Prevalence of Pneumocystis jirovecii Pneumonia Amongst the Non-HIV Immunosuppressed
}

\author{
Zachary Jones, MD \\ Thomas Jefferson University, zachary.jones@jefferson.edu \\ Anusha Ganesh, MD \\ Thomas Jefferson University, anusha.ganesh@jefferson.edu
}

Follow this and additional works at: https://jdc.jefferson.edu/tmf

Part of the Medicine and Health Sciences Commons

Let us know how access to this document benefits you

\author{
Recommended Citation \\ Jones, MD, Zachary and Ganesh, MD, Anusha (2015) "Rising Prevalence of Pneumocystis jirovecii \\ Pneumonia Amongst the Non-HIV Immunosuppressed," The Medicine Forum: Vol. 16 , Article 14. \\ DOI: https://doi.org/10.29046/TMF.016.1.013 \\ Available at: https://jdc.jefferson.edu/tmf/vol16/iss1/14
}

This Article is brought to you for free and open access by the Jefferson Digital Commons. The Jefferson Digital Commons is a service of Thomas Jefferson University's Center for Teaching and Learning (CTL). The Commons is a showcase for Jefferson books and journals, peer-reviewed scholarly publications, unique historical collections from the University archives, and teaching tools. The Jefferson Digital Commons allows researchers and interested readers anywhere in the world to learn about and keep up to date with Jefferson scholarship. This article has been accepted for inclusion in The Medicine Forum by an authorized administrator of the Jefferson Digital Commons. For more information, please contact: JeffersonDigitalCommons@jefferson.edu. 


\title{
Rising Prevalence of Pneumocystis jirovecii Pneumonia Amongst the Non-HIV Immunosuppressed
}

\author{
Zachary Jones, MD and Anusha Ganesh, MD
}

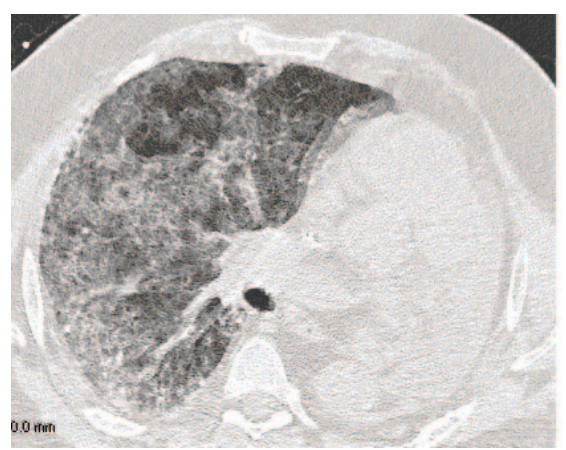

Figure 1. Cross Section CT scan with ground glass opacities, severe interlobar septal thickening likely related to interstitial pneumonia and stable postoperative changes following left pneumonectomy from day 1 of hospitalization.

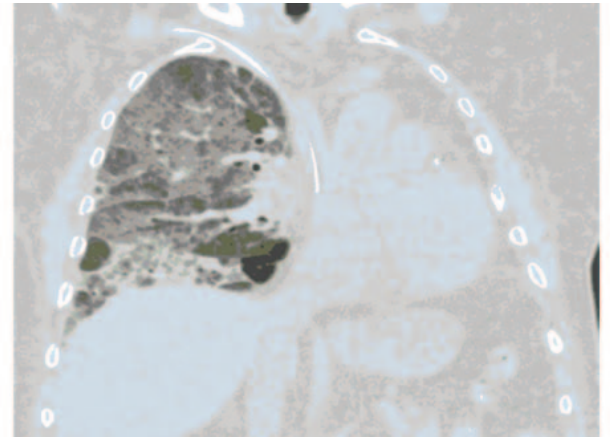

Figure 2. Coronal Cut of CT scan demonstrating severe interstitial pneumonia cut from day 9 of hospitalization.



Figure 3. Sagittal cut from CT scan demonstrating interstitial pneumonia from day 9 of hospitalization.

\section{INTRODUCTION}

Fungal Pneumonia caused by Pnemocystis Jirovecci (PCP) has long been associated with morbidity and mortality in HIV-positive patients. With the widespread use of high dose corticosteroids and biologic therapies, the prevalence of PCP infection in the non-HIV immunosuppressed population has increased significantly. A lack of formalized prophylaxis guidelines in these specific populations has lead to increasing rates of preventable infection and death.

\section{CASE PRESENTATION}

The patient is a 63-year-old man, admitted to the hospital for persistent shortness of breath following a seven-day course of Levofloxacin taken as an outpatient. Two months prior, the patient was found by his hematologist to have a hemoglobin level of $5.5 \mathrm{~g} /$ $\mathrm{dL}$, and was diagnosed with autoimmune hemolytic anemia. He was started on a 12 week Prednisone taper following an initial blood transfusion. Three weeks prior to admission, the patient began to develop shortness of breath worse with ambulation, a non-productive cough, and he denied fevers. At time of admission, the patient was taking Prednisone $40 \mathrm{mg}$ daily.
The patient's past medical history was significant for Hodgkin's Lymphoma in 2001 treated with Adriamycin, Bleomycin, Vinblastine and Dacarbazine (ABVD) and radiation therapy, with recurrence in the nasopharanx and lung requiring further ABVD cycles and left pneumonectomy in 2005. Patient also had renal cell carcinoma requiring nephrectomy in 2007. Social history was significant for a 5-year pack history of cigarette smoking. Family history was significant for myocardial infarction in his father at age 69.

Vital signs at presentation were temperature of $99.4 \mathrm{~F}$, blood pressure of 157/70, pulse of 105 beats per minute and oxygen saturation of $90 \%$ on room air. Pertinent positive physical exam findings were sinus tachycardia and diffuse wheezing in all right lung fields and lack of breath sounds on the left. Patient was resting comfortably and able to answer questions without distress. On admission, patient had a normal white blood cell count and a hemoglobin level of $9.8 \mathrm{~g} / \mathrm{dL}$. Computed tomography scan of the thorax showed mild diffuse, nonspecific ground glass opacities. 


\section{The Medicine Forum, Vol. 16 [2015], Art. 14}

\section{DIFFERENTIAL DIAGNOSIS}

The differential diagnoses for shortness of breath in this specific patient with previously treated hematologic malignancy currently on chronic steroids is vast, including pulmonary infection, Chronic Obstructive Pulmonary Disease (COPD), or volume overload. Also, two components of ABVD regimen can cause side effects producing symptoms of shortness of breath: Adriamycin can cause cardiomyopathy and subsequent congestive heart failure, and Bleomycin can cause pulmonary fibrosis. Further possible diagnoses include pulmonary embolism and opportunistic infection given his steroid use.

\section{OUTCOME}

The patient was initially started on Moxifloxacin and given Furosemide intravenously. The patient's Ventilation/Perfusion scan was negative for embolism, echocardiogram revealed no systolic dysfunction, and HIV antibody, Antinuclear antibodies, perinuclear anti-neutrophil cytoplasmic antibodies ( $p$-ANCA), cytoplasmic-ANCA (c-ANCA) and rheumatoid factor were negative. The patient's respiratory viral and sputum culture were also negative. A right heart catheterization completed on day 6 of hospitalization revealed pulmonary hypertension. Bronchoscopy was performed on day 10 due to worsening hypoxia and worsening radiographic evidence of interstitial process on antibiotics and diuretics (Figure 1,2,3). Bronchoalveolar lavage demonstrated presence of Pneumocystis jirovecii.

The patient was started on Trimethoprim/ Sulfamethoxazole (Bactrim). On day 15, the patient required continuous Bilevel Positive Airway Pressure (BIPAP) for worsening hypoxia and was transferred to the Intensive Care Unit. The patient was transitioned to intravenous Bactrim and his Prednisone dose was increased. On day 23, the patient required intubation and suffered pulseless electrical activity and return of spontaneous circulation was not achieved after 20 minutes of cardiopulmonary resuscitation.

\section{DISCUSSION}

Pneumocystis pneumonia (PCP) is a life threatening disease caused by the fungus Pneumocystis jirovecii previously classified as Pneumocystis carinii. While PCP is historically associated with HIV and CD4 counts less than $200 / \mu \mathrm{L}$, there have been increasing case reports in HIV negative patients treated with high dose steroids, Methotrexate, Tacrolimus, Infliximab and Etanercept. In the HIV negative population, PCP has a higher likelihood of causing fulminant pulmonary disease more likely requiring mechanical ventilation.

In a study looking at those with Adult T Cell Leukemia treated with corticosteroids, there was a $16.6 \%$ overall risk of PCP infection without prophylaxis. ${ }^{3}$ In a small case series of patients with Giant Cell Arteritis treated with high dose Prednisone, $85 \%$ presented on admission solely with dyspnea, $43 \%$ required mechanical ventilation, and $29 \%$ died. ${ }^{4}$ The documented PCP mortality rate ranges from $51 \%-80 \%$ in HIV negative patients. ${ }^{1}$ In a review of neurology literature, up to $90 \%$ of patients were treated with high dose corticosteroids prior to diagnosis with PCP. ${ }^{6}$ In a Cochrane review of immunocompromised patients without HIV, thirteen trials showed a reduction of $85 \%$ in incidence of PCP when prophylaxis was administered. ${ }^{6}$ Across multiple specialties, no firm prophylaxis guidelines have been set. It is generally accepted that any patient on greater than or equal to Prednisone $20 \mathrm{mg}$ per day for greater than 4 weeks should be considered for PCP prophylaxis with Bactrim. There was found to be no statistically significant difference when comparing once daily versus three times weekly dosing of Bactrim for prophylaxis. ${ }^{\circ}$

The diagnosis of PCP can be delayed due to nonspecific radiographic findings and vague symptoms at time of presentation, as seen with our patient who was not diagnosed until day 10 of hospitalization. Novel serologic markers that detect PCP have been described, with B-1,3 Glucan approaching 100\% sensitivity but lacking specificity with regards to detecting PCP. Another marker, KL-6, is a glycoprotein elevated in interstitial pneumonitis caused by PCP, which is elevated in the HIV population, but has not been shown to reliably detect the disease in non-HIV patients. ${ }^{2}$ In the non-HIV immunosuppressed, the mainstay of diagnosis involves direct sampling via bronchoalveolar lavage and should be considered early in hospital stay for any patient at risk for PCP. 
Once a diagnosis is made, PCP treatment is similar regardless of reason for immunosuppression. Bactrim is the gold standard of therapy with Dapsone, Atovoquone, or Pentamidine used when Bactrim cannot be tolerated? Duration of treatment is not well established in non-HIV patients but should extend to at least 14 days in contrast to the minimum of 21 days for the HIV positive. In patients with HIV and an A-a gradient greater than $35 \mathrm{mmHg}$ or a $\mathrm{PaO} 2$ less than $70 \mathrm{mmHg}$, corticosteroids should given. In contrast, adjunctive high dose corticosteroids increased mortality in the non-HIV immunocompromised and are not recommended.

\section{KEY POINTS}

Pneumocystis pneumonia is a devastating disease of both HIV and non-HIV patients alike. While there are no formalized guidelines, any patient on a corticosteroid dose greater or equal to Prednisone $20 \mathrm{mg}$ per day for more than 4 weeks should be on prophylaxis. In doing so, the rates of PCP can be drastically lowered in these patient populations preventing undue burden of disease.

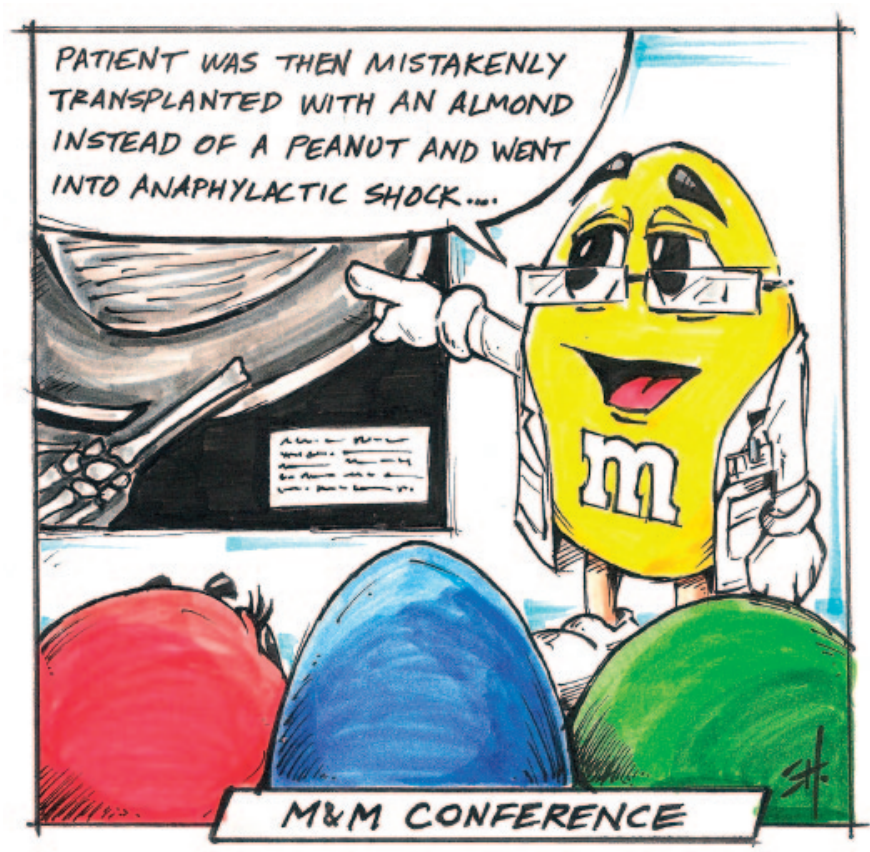

\section{REFERENCES}

1. Mori $S$ and Sugimoto M. Pneumocystis jirovecii infection: an emerging threat to patients with rheumatoid arthritis. Rheumatology (Oxford). 2012 Dec;51 (12):2120-30.

2. 205448920. Eddens $T$ and Kolls JK. Pathological and protective immunity to Pneumocystis infection. Seminars in Immunopathology. 2014 Nov 25. Epub ahead of print.

3. 205449016. Maeda T, Babazono A et al. Quantification of the effect of chemotherapy and steroids on risk of Pneumocystis jiroveci among hospitalized patients with adult T-cell leukaemia. British Journal of Haematology. 2014 September 30. Epub ahead of Print.

4. 205449112. Kermani TA Ytterberg SR, et al. Pneumocystis jiroveci pneumonia in giant cell arteritis: A case series. Arthritis Carre Res (Hoboken). 2011 May; 63 (5): 761-5.

5. 205449208. Kelly DM and Cronin S. PCP prophylaxis with use of corticosteroids by neurologists. Practical Neurology. 2014 April; 14(2):74-6.

6. 205449304. Stern A, Green H et al. Prophylaxis for Pneumocystis pneumonia (PCP) in non-HIV immunocompromised patients. Cochrane Database Systems Review 2014 Oct 1;10. CD005590.

7. 205449400. Crozier F. Pneumocystis carinii pneumonia prophylaxis: current therapies and recommendations. Journal of Pediatric Oncology Nursing. 2011 May-June; 28 (3): 179-84.

8. 205449496. Lemiale V, Debrumetz A et al. Adjunctive steroid in HIV-negative patients with severe Pneumocystis pneumonia. Respiratory Research. 2013 Aug 28; 14:87. 